\title{
Acercamiento transdisciplinario a las realidades complejas e interconectadas a partir de la teoria general de sistemas ${ }^{1}$
}

\author{
Ingrid Nederr Donaire ${ }^{2}$
}

Resumen

El concepto de sistemas tiene gran vigencia en la actualidad, y no solo se utiliza en el ámbito de la investigación sino que también se destaca en diversas áreas y campos que definen el comportamiento social, económico, político, tecnol ógico y cultural. Desde finales de los sesenta este concepto habia invadido los campos de la ciencia, el pensamiento, el habla popular y los medios de comunicación de masas, a tal punto que existen ocupaciones y profesiones con su nombre, por ejemplo: Sistemas de información, Ingeniería de sistemas y Sistema educativo. Ellos pueden definirse de manera general como un conjunto complejo de elementos relacionados cuyo proposito común, en un plano más abstracto, es movilizar la convergencia de saberes y promover la tarea inter y transdisciplinaria. Intrínseca en estos argumentos está la intencionalidad de este artículo que presenta mediante una hermenéutica profunda, argumentos teóricos que emergen de la comprensión de la Teoría General de Sistemas desde sus enfoques, antecedentes y bases teológicas, epistemicas y ontologicas. Tambien se consideran los principales aspectos en torno a su historia y lo que esta representa en la contemporaneidad, especialmente para poder transcender hacia la interrelacion de diversas áreas del saber, mediante el diálogo entre las ciencias y lo que esto supone

1 Articulo de Reflexión sobre Trabajo de Investigación Presentado ante la Universidad Nacional Experimental de las Fuerza Armada Nacional. UNEFA. Doctorado en Ciencias Gerenciales. Línea de Investigación Gerencia y Transformación Estratégica. Caracas Venezuela. Abril 2009.

2 Doctora en Ciencias de la Educación. UNERG (2007) y Doctora en Ciencias Gerenciales. UNEFA (2009). Actualmente Docente Investigador con la Categoría de Asociado en el Area de Ingeniería de Sistemas de la Universidad Nacional Experimental "Rómulo Gallegos" (UNERG), ubicada en San Juan de los Morros. Estado Guárico. Facilitadora a Nivel Doctoral en ambas Universidades. M iembro de la Red de Investigadores sobre la Transcomplejidad. REDIT. Correo electrónico: inederr@gmail.com, inederr@cantv.net. Twitter: @inederr 


\title{
IngidNederLonaire
}

en el discurrir de configuraciones multidimensionales y heterogéneas que privilegian las tramas configurativas en red. Desde esta perspectiva se esboza el cometido de esta reflexión cuyo recorrido se fundamenta en las ciencias de la compleiidad, la cibernetica de primero y segundo orden, la comunicacion orquestal de la Escuela de Palo Alto y los principios para un pensar complejo y autopoiético, argumentos que permiten captar las Realidades Complejas e Interconectadas.

Palablaras clave: Sistemas, transdisciplinariedad, realidades complejas e interconectadas.

\section{A transdisciplinary approach to complex and interconnected realities based on the general system theory}

\begin{abstract}
The concept of system has great validity today, not only in the field of research but also and mainly in various areas and fields that define social, economic, political, technological and cultural behavior. Since 1968, Bertalanffy was aware of these issues to the extent that there were occupations and professions with such a name, eg: Information Systems, Complex Systems and Educational System, among others, which can generally be defined as a complex set of related elements with the common purpose to mobilize the convergence of knowledge and promote inter- and trans- disciplinary work. Immersed in these arguments, this article intends to show by a deep hermeneusis, theoretical arguments that emerge from the understanding of the General Systems Theory from their approaches, backgrounds, their theological, epistemic and ontological grounds as well as key issues about its history and its meaning in contemporary world, especially to transcend towards linking and interrelation of different areas of knowledge through the application of dialogue between sciences as a path to inter- and transdisciplinarity and the implications in the flow of multidimensional and heterogeneous configurations favoring network configurative frames. This reflection departs from such a perspective, and moves forward through a path founded in the sciences of complexity, first and second rank cybernetics, orchestral communication at Palo A Ito Unified School, and the fundamentals of complex and auto-poietic thinking, all of which allows us perceive complex interconnected realities
\end{abstract}


Revista de

investigaciones UNAD

Volumen 10. Número 1. Junio 2011

Key wards: Systems, complex and interconnected realities, transdisciplinarity.

Recibido: 17 Febrero de 2011

Aceptado: 26 Mayo de 2011

Relevancia de la Teoría general de Sistemas en el acercamiento trandisciplinario de las realidades complejas interconectadas

En la reflexión que se genera en la comprensión de la Teoría General de los Sistemas (TGS) y sus diferentes enfoques, asI como lo que estos conllevan en el acercamiento al dialogo de saberes que privilegia lo inter y transdisciplinario como algo natural y necesario, especialmente cuando se pretende el abordaje de reali dades complejas, multidimensionales e interconectadas, se destaca una complementariedad de momentos sucesos, eventos y circunstancias que se integran en dos vertientes de significacion.

Por un lado, se muestra cómo surge la TGS bajo concepciones neopositivistas, ancladas en el Funcionalismo-Estructuralismo, como corrientes de pensamiento que se nutren mutuamente, y que surgen en la Europa de post querra, trascendiendo progresivamente hasta alcanzar su auge y desarrollo en Estados Unidos, como respuesta a la depresión y los desacierttos que suponían os enfoques cientificos y humanistas desde los que se percibian y manejaban las organizaciones en esa epoca.

Es por ello que inicialmente esta perspectiva hace eco a la objetividad y separabili idad de la reali idad obj eto de estudio, concediendo importancia a la refinacion del conocimiento para garantizar su integridad y eliminar cualquier vestigio de subjetividad. Por esta razon, util iza la estructura como interrelación de partes, elementos y funciones que lineal y jerrárquicamente dependen de un "Todo Superior" que perfilaba su homogenización.

Por otro lado, se traza un recorrido donde se percibe la integración de concepciones, ideas, principios y pensamientos que permiten formular teorías o metateorias de caracter general para comprender y explicar la infinidad de relaciones que surgen en cualquier realidad donde se co-construye el conocimiento en un ir y venir de aconteceres y circunstancias.

Ante estass vertientes de significación cabe preguntarse lo siguiente: iEn qué concepcion o perspectiva nos ubicamos?, ¿Como lograr la coexistencia de estas 


\section{IngidNeder Donaire}

Acercamiento transdisciplinario a las realidades complejas e interconectadas a partir de la teoria general de sistemas,

artículo de reflexión

perspectivas?, ¿Cuándo y cómo podemos distinguir los diferentes enfoques que se involucran en ello?, ¿Que tiene que ver la TGS en la percepcion, comprension e interrelación de las realidades complejas e interconectadas? iRelacionamos lineal y jerárquicamente el todo y las partes, o por el contrario, ¿Privilegiamos la heterogeneidad, multidimensionalidad e interdependencia de cual quier acontecer? Para dar respuesta a estos cuestionamientos, surgen los siguientes propositos:

Comprender el devenir sistémico desde el contexto histórico-social de la humanidad.

Interpretar la relación que existe entre sistemas, comunicación orquestal, cibernetica, procesos de autoorganización biologica y principios para un pensar complejo en la configuracion de tramas interconectadas.

Generar una trama teórico-conceptual para el acercamiento transdisciplinario a las realidades complejas e interconectadas.

Este estudio se muestra altamente significativo, ya que presenta la posibilidad de vislumbrar, los principios y pedestales conceptuales que permiten la percepcion, comprensión e intepretacion de las realidades complejas e interconectadas.

El devenir inter y transdisciplinario a partir de la Teoría General de Sistemas

Los sucesos, circunstancias y acciones que inciden en el quehacer cotidiano del mundo contemporaneo, condicionan a la sociedad, las organizaciones, los grupos y los individuos a transformaciones continuas que se manifiestan por altos márgenes de incertidumbre y ambiguedad. Desde este contexto se perfila la comprension del devenir de cual quier realidad, ya sea social, educativiva, politica, tecnologica o cultural, las cuales se encuentran intrinsecamente vinculadas en una trama de interacciones e interrelaciones que emergen de manera natural como autonomias y dependencias entre sus elementos constituyentes, reccreanndo una multidiensionalidad interconectada que requiere un giro onto-epistemico que empieza por asumir lo inter y transdisciplinario, para poder abordarla, comprenderla e interpretarla.

Sin embargo, pasará mucho tiempo antes de que la humanidad se apropie de estos aspectos, ya que durante durante muchos años asumio cosmovisiones fundadas en el mecanicismo, matematicismo y organicismo, en una dialectica que marchaba de los descubrimientos e inventos de la ciencia natural, a las ciencias sociales, todas ellas atravesadas, de alguna manera, por el enciclopedismo, el romanticismo, la religión, la metafísica y el sentido común, constituyendo un velo que fragmentaba todà realidad.

Los șistemas se refieren a un todo o conjunto complejo de partes y elementos relacionados que buscan un objetivo comun. En tal sentido, sus antecedentes 
Revista de

investigaciones UNAD

Volumen 10. Número 1. Junio 2011

presentan un horizonte que se remonta a 2500 años $A_{\text {. }} C_{1,}$ durante la cultura china, la cual focaliza y guia su existir y convivir mediante la concepción de un todo organizado gracias a la relacion de cuatro elementos basicos como son: el fuego, el aire, la tierra y el agua.

De igual manera, según Konemman (2005), los Presocráticos, 650-500 A. $C$., dan cuenta de este supuesto cuando en el intento de conseguir la esencia de las cosas y la comprension del universo, recurren a la totalidad del mundo mediante la relacion de los opuestos, basados en la contemplacion empirica de la multiplicidad de realidades. En este sentido, Heraclito (VI A. C.) plantea el origen del conocimiento mediante la relacion de dos principios: Todo fluye y nada permanece, y la unidad de los contrarios. Para ello esgrime la analogía de un río, aduciendo que nadie se baña dos veces en el mismo.

En la época de Platón y A ristóteles surge esta máxima: "El todo es más que la suma simple de las partes". Se considera a A ristoteles como el, primero en considerar la dinamica de los sistemas. Sin embargo, la filosofía clásica y sus pensadores fundaron su concepcion en la nocion de orden de elementos secundarios en torno a uno primario o principal. Todo este orden se funda en dos clases de causas esenciales, las intrínsecas y las extrinnsecas. A las primeras corresponden la Logica o Causalidad Formal y la Holologica o Causalidad Material, y a las extrínsecas pertenecen la Etiologica o Causalidad Eficiente y la Teleológica o Causalidad Final.

En la E dad M edia sepresenta una unilaterali dad de los métodos de la investigación científica que se identifican con los de la Fisica y la Matemática.

En el siglo XIX surge un movimiento filosófico y científico que intenta poner remedio a esta linealidad analíicca de la ciencia. Se destaca Dilthey y su Globus Intelectus, como cultivo de las ciencias para las obras del hombre.

Es en el transcurso de la segunda década del siglo XX, cuando ciertos acontecimientos y aportes en el mundo de la ciencia hicieron que estas tendencias comenzaran a cambiar. Tal es el caso del Principio de la Relatividad de Albert Einstein (1879-1955), que trata la física del movimiento de los cuerpos en ausencia de fuerzas gravitatorias.

Según Sears y Zemanski (1971), esta teoría consiste en que dos observadores que se mueven rel ativamente uno al lado del otro, con una velocidad cercana a la de la luz, a menudo mediran diferentes interval os de tiempo y espacio para describir las mismas series de eventos. En otras palabras, la percepcion del espacio y el tiempo depende del estado de movimiento del observador. Esto es posible por la aceptación de la teoría de los cuantos, ya que al admitir que los componentes fundamentales de la materia son espacio-temporales, se consiente que si una de las dimensiones atada a ellos se alarga, la otra debe acortarse. 


\section{IngidNederDonaire}

Acercamiento transdisciplinario a las realidades complejas e interconectadas a partir de la teoria general de sistemas,

artículo de reflexión

Por otro lado, Neils Bohr (1885-1962), conel Principio de Correspondencia abre la Mecanica del Quantum, tambien conocida como Mecánica ondulatoria, que rompe con el paradigma newtoniano de la física establecido hasta ese entonces.

Con ella se descubre que el mundo atómico no se comporta como partículas indivisibles, sino que presenta la dualidad onda-partícula, diciendo, que un mismo fenomeno puede tener dos percepciones distintas, ser corpúsculo y onda a la vez.

Con esta teoría seintroducen los conceptos de incertidumbre, indeterminación o cuantizacion Asl, al ser imposible fijar a la vez la posicion y el momento de una partícula, se renuncia al concepto de trayectoria, que es vital en la mecanica clasica.

Estos aportes incitan al mundo de la ciencia, a que el conocimiento de la realidad se entienda como al go mas complicado, pero a pesar del quebrantamiento del paradigma newtoniano de la fisica, la concepcion de dualidad onda-particula y la introducción de los conceptos de incertidumbre e indeterminacion, la cosmovision hegemónica y lineal del positivismo se ha extendido hasta la actualidad, intentando hacer creer que la naturaleza y la sociedad pueden ser ordenadas para el progreso y desarroll 0 . Desde entonces se ha venido configurando una concepción que admite que los procesos naturales se interrelacionan entre sí y se mueven del orden al desorden, lo cual ayuda a comprender la dimensionalidad compleja de as realidades interconectadas, que a primera vista parecen ser inextricables y dificiles de abordar.

Es por ello que se demanda una nueva forma de enfrentar los fenómenos naturales y sociales, y que la comunidad científica a mediados de los noyenta comienza a entender los fenómenos de esta manera. Tal como se destaco, los defensores del movimiento determinista consideraban más práctico observar los fenomenos de forma analitica, estudiando las partes como si fuesen independientes de su totali idad, y cayeron en un reduccionismo que sego por mucho tiempo la compl ejidad presente de todo ser vivo, ya que la unidad mas pequena es tan compleja como la totalidad del conjunto.

De aquí parte el desplome de la concepción reduccionista y mecanicista, más aún cuando este esquema resul taba insuficiente para enfrentarse a los problemas teoricos y practicos planteados por la tecnologia. Debido a ello, se comienza a tomar en cuenta las relaciones entre los componentes de las realidades, Io cual reeoriento el pensamiento científico hacia esta perspectiva, especialmente para vislumbrar en la trama de sus interacciones, la complejidad de las totalidades. Estos aspectos hacen que Von Bertal anffy (1968) enuncie un cuerpo de ideas denominado Teoria General de los Sistemas. 
Revista de

investigaciones UNAD

Volumen 10. Número 1. Junio 2011

Esta teoría constituye un conjunto de postulados que se fueron afinando progresivamente con su aplicacion, a partir de los cuales se desprende que cualquier fenomeno de la reali dad debe ser considerado en terminos de sistemas, es decir, por las relaciones e interacciones de sus componentes, "por dificil y fluctuante que sea, la definición de entidades socioculturales" (p. 6). Por ello este autor intento favorecer, la tarea interdisciplinaria, para lograr mayor comunicacion, integración y unidad en la ciencia. De esta manera, presenta un marco conceptual y teorico que reivindica las propiedades intrinsecas de la dinamica sociocultural y su complejidad.

En correspondencia con las particularidades y propiedades de la realidad, y con su interrelación el autor esboza una serie de principios y leves aplicables a mas de uno de los comportamientos tradicionales del conocimiento, lo cual impulso desde tres vertientes significativas como son: la investigacion de isomorfismos de conceptos, leyes y modelos en varios campos del saber;, su transferencia de un campo de conocimiento a otro, sin importar su genero particular, ni la naturaleza de sus elementos componentes, ni las relaciones o fuerzas que imperen entre ellos; y finalmente, la unificación de criterios cientificos mediante la comunicacion de las disciplinas del saber.

Según Von Bertalanffy, el isomorfísmo o correspondencia entre los modelos permite ap licar las mismas caracteristic cas a las más variadas disciplinas, ya que existen "correspondencias entre los principios que rigen el comportamiento de entidades que son intrínsecamente muy distintas" (p. 33).

Del hilo axiomático anterior, el autor define los sistemas como complejos elementos en interacción, enfatizando en el hecho de que conceptos, modelos y leves emergen una y otra vez en campos muy diversos, instituil dos en realidades diferentes, de las cual es en muchos casos emergen principios semejantes- Sin embargo, el isomorfismo es más que analogias vagas y superficiales, ya que asi como existen las similitudes, las diferencias tambien son constatables, y por ello, establece una distincion entre sistemas cerrados y abiertos, considerando a los sistemas cerrados como aislados del medio circundante, y a los abiertos, sometidos al intercambio de materia, energía e informacion con el entorno, como los sistemas vivientes.

Con base en la distinción que hace en cuanto a los sistemas abiertos y cerrados, Von Bertalanffy, toma de Walter Cannon (1871-1945), el concepto de Homeostasis psicológica o equilibrio dinámico entre entradas y salidas, que permite cambios continuos en el sistema, con predominio de condiciones relativamente uniformes, es decir, la tendencia general de todo organismo al restablecimiento del equilibrio interno cada vez que este es al terado. Por ello, en los sistemas cerrados existe una continua tendencia hacia la desorganización y destrucción del orden, en contraposicion a los los sistemas vivientes que presentan una tendencia hacia niveles de mayor heterogeneidad y organizacion. 


\section{IngidNeder Donaire}

Acercamiento transdisciplinario a las realidades complejas e interconectadas a partir de la teoria general de sistemas,

artículo de reflexión

De igual manerac, Shannon y Weaver (1949) incorporan de la teoría matemática de la comunicación el concepto de Información y entropla negativa, fundamentado en que la información constituye la noción general de esta teoría, y su distribución es considerada como la respuesta a diversas alternativas que le dan apertura a la entropia negativa desde su concepcion como medida de desorden. De aqui que la entropia negativa o informacion sea una tendencia hacia el orden u organizacion. Otro concepto central de esta teoria corresponde a la retroalimentación, percibida como mecanismos homeostaticos de regulacion o mantenimiento del equilibrio en la organizacion del sistema. A partir de las nociones de adaptabilidad, intencionalidad y. persecucion de metass, considera el comportamiento teleologico de los sistemas vivos como algo definible en terminos cientificos.

Otro aspecto que cobra fuerza y es relevante en la teoría general de los sistemas, lo consituye el hecho de que los sistemas abiertos al canzan estados finales, de donde emerge el principio de equifinalidad, que Von B ertalanffy define como la propiedad de al canzar "el mismo estado final partiendo de diferentes condiciones iniciales y por diferentes caminos" (p. 40). Fundamentado en esta premișa y en la interaccion dinamica de los componentes, utiliza la nocion de organizacion como elemento importante para comprender la comple ejidad de los sistemas vivos, y como estos se mantienen en un equilibrio dinamico, toma de la cibernética el concepto de Retroalimentación, unido a mecanismos de control, positivos y negativos, que amplifican y corrigen la desviacion respectivamente.

Sistemas, comunicación, cibernética, autopoiesis y complejidad para vislumbrar las realidades complejas interconectadas

Según Jutoran (1994), a lo largo de los años 50 y 60 también hubo una fuerte infiluencia de la teoría de la comunicacion, proveniente de un grupo de importantes investigadores de distintos campos de conocimiento (psiquilatria, antropologia, sociologia, psicología y lingüistica), que darian cuerpo a lo que se conocería como "L a Escuela de Palo Alto", tambien conocida como U niversidad invisible. Este grupo concedio importancia a los contextos, la eficacia de la informacion, la comunicacion no verbal, la metacomunicacion y las pautas que la conectan, caracteristicas que tal como lo enuncia,Wazlawick (1990), uno de sus exponentes, ayudan a comprender que todo fenomeno se comporta de forma reciproca e interdependiente con su tejido vivencial.

El argumento anterior se sustenta en que este grupo de investigadores comparte el criterio de Winkin (1984), en cuanto a la comunicacion como "proceso social permanente que integra múltiples modos de comportamiento, la palabra, el gesto, la mirada, la mimica, el espacio interindividual, etc." ( $p, 32)$, considerandola de este modo, como un todo integrado regido por un conjunto de codigos y reglas determinadas por cada cul tura, como el modelo tel egráfico de Shannon y Weaver, que, consideraba la comunicacion como intercambio o transmisión de información de forma lineal, concepción que este autor ha 
Revista de

investigaciones UNAD

Volumen 10. Número 1. Junio 2011

ampliado y configurado como un modelo orquestal, en el que se considera la realidad social en la cual se encuentra inmerso.

A tono con la teoría sistémica y su devenir en la Escuela de Palo Alto, G regory Bateson (1904-1980), presenta sus argumentos, en cuanto a la pauta que conecta a todos los seres vivientes. Esto se refiere a las configuraciones, las formas y las relaciones que pueden ser observadas en todos los fenomenos. Bateson.(1976) propuso la nocion de Contexto como elemento fundamental de toda comunicacion y significacion, planteando que no se debe ais lar el fenomeno de su contexto, pues cada fenómeno tiene sentido y significado dentro del ambito en que se produce.

I gualmente propone que en el mundo de las formas vivientes es necesario tomar en cuenta conceptos tales como información y relación, para lo cual es fundamental encontrar un nuevo lenguaje que permita describir la recursividad de todos los elementos que se mueven conjuntamente en un proceso.

Casi concurrentemente a la teoría de sistemas, se desarrolló la cibernética, planteada por Wiener en 1948, como la ciencia de la organizacion, la comunicacion y el control. Segun W iener (2003), la cibernetica se basa en la concepcion de que no es posible considerar una parte del fenomeno separada de las otras, debido a su interdependencia. Por ello, surge el estudio de los mecanismos de regulación mediante la retroalimentacion, la cual se fundamenta en la teleologla de la circularidad para mantener un equilibrio dinamico.

Ahora bien, la historia de la cibernética se puede observar como un proceso que se desarrolla en tres niveles de compleiidad, tomando en cuenta las consideraciones de Von Foerster (1911-2002). Estos son; Cibernética de "cero orden", implicita;' cibernetica de "primer orden", explicita y cibernetica de "segundo orden", reflexion sobre la reflexion de la cibernetica, resultando imposible acceder a un nivel superior a este, dado que en opinion de Maturana y Foerster (1988), cuando se reflexiona sobre la reflexión se cierra el circulo de argumentacion y se produce una clausura organizacional que solo puede trascenderse a si misma dentro de sí misma.

En los estudios de epistemología sistémica hechos por Jutoran, se hace referencia a 10 anteriormente planteado y se aborda este devenir descle e primero que inventó un sistema cibernetico, Heron de A lejandría en el año 62 a. C. cuando nace la cibernetica de cero orden. Este sistema consistía en un mecanismo de retroal imentacion negativa que regul aba el liquido que sal ia de una jarra al Il enar un vaso, y se detenia en un determinado momento previsto anteriormente.

La cibernética de primer orden nace con Norbert Wiener entre 1954 y 1967, el cual en sus estudios de las trayectorias que siguen los disparos de los canones antiaereos, utilizo el principio de retroalimentácion o feedback, para optimizar su objetivo mediante la reintroduccion de los resultados del desempeño del 


\section{IngidNeder Donaire}

Acercamiento transdisciplinario a las realidades complejas e interconectadas a partir de la teoria general de sistemas,

artículo de reflexión

sistema. De esta manera, los efectos retroactúan sobre las causas, convirtiendo el proceso lineal en circular.

En esta etapa se consideraba que los procesos de feedback negativo permitían mantener la organizacion y que los procesos de ampliacion de la desviacion llevaban a la desorganizacion, el caos y la destruccion del sistema. Por lo tanto, la homeostasis de Cannon (1936) como proceso autocorrectivo, era el concepto predominante.

Siquiendo con el proceso de desarrollo de la cibernética, el tercer orden fue definido por M aruyana (1974) como segunda cibernética, bajo la consideración de que todo sistema viviente depende para su supervivencia de dos procesos: "morfostasis" que se refiere al mantenimiento de un sistema constante a traves de mecanismos de retroalimentación negativa, y "morfogenesis", la desviacion y variabilidad del sistema a traves de mecanismos de retroalimentacion positiva.

De acuerdo con estos argumentos, mediante los trabajos del físico llya Prigogine (1917-2003), referentes al orden a partir de la fluctuacion, se considera que los procesos que promueven el desorden y la desorganizacion no necesariamente son destructivos, ya que si se mantienen las fluctuaciones y no son contrarrestadas por mecanismos correctores, seproduce una bifurcacion que genera un salto cual itațivo hacia una nueva organizacion. En este desarrollo del pensamiento cibernetico, la ampliacion de la desviacion y los mecanismos de retroalimentacion positiva adquieren un nuevo status, puesto que son considerados procesos favorecedores del cambio, esenciales para la evolucion de los sistemas vivientes.

En 1972, Foerster sugiere cambiar este nombre por "Cibernética de segundo orden" o "Cibernética de los sistemas observantes". Esto surge debido a que la epistemología tradicional considera que la realidad existe independientemente de quien la observa; el pensamiento científico determinista se basa en esta premisa al considerar que la objetividad en la ciencia es fundamental y que, por lo tanto, las propiedades del observador no deben estar incluídas en lá descripción de sus observaciones.

Sin embargo, Foerster (1988) trae a colación dos aspectos significativos: Primeramente, el principio de relatividad de Einstein, en cuanto a que las observaciones no son absolutas sino relativas desde el punto de vista de observador, es decir, segun su sistema de coordenadas. En segundo lugar, en el acto de observar se influye tanto sobre el objeto observado, que se anula toda expectativa de prediccion por parte del observador, convirtiendo la incertidumbre y la indeterminacion, en absolutas.

Por ello, enfatiza que los requisitos claves para abordar la epistemología de los sistemas vivientes son: la reintroducción del observador en lo observado, la perdida de la neutralidad y la objetividad, recurriendo para ello a la circularidad 
Revista de

investigaciones UNAD

Volumen 10. Número 1. Junio 2011

dinámica de la retroalimentación y las nociones inmersas en el concepto de recursividad, así como a las paradojas, la causal idad recíproca, la autorreferencia, la autoorganizacion, la complejidad y la autonomía.

Con base en lo antes planteado, se presenta una cibernética de la cibernética, al considerar la observacion del observador, ya que el objeto de estudio pasa a constituirse en el observador, observando su observacion, razon por la cual se llama cibernética de segundo orden. De esta manera, desde la perspectiva de la cibernetica de segundo orden se considera que existe, "un continuo proceso circular y repetitivo en el que la epistemologia determina lo que vemos; esto establece lo que hacemos; a la vez nuestras acciones organizan lo que sucede en nuestro mundo, que luego determina nuestra epistemologia" Foerster (1991, p. 28).

Otra importante contribución a la comprensión de las relaciones e interacciones humanas la constituyen los trabajos de Humberto Maturana con la colaboracion de Francisco Varela (1946-2001), donde presentan, por los años sesenta, SU propuesta de que los seres vivos se constituyen y operan como sistemas cerrados de producciones moleculares, y abiertos al flujo material y energetico.

M aturana (1990) acuña el término autopoiesis para referirse a la organización que define y constituye a los seres vivos. De esta manera la presenta como una unicad compuesta, cuya organizacion puede ser descrita como una red de produccion de componentes. I gualmente expone que el mundo se crea en el lenguaje y en el diálogo que remiten a la ética en la convivencia y que tiene fundamentales repercusiones en el devenir de las relaciones humanas.

Niklas Luhmann (1998) y Edgar Morin (1993) han realizado intentos para articular las ciencias humanas con las teorías de la información, las de los sistemas, la cibernética y los procesos de autoorganizacion biológica, con la finalidad de construir una estrategia metodologica que trate de estar a l a altura de la complejidad que implica reconocer en el mundo la dialogica de la vida social.

Descle esta perspectiva, la comunicación es considerada la base de la interacción social, y como tal, es el principio básico de la sociedad, su esencia. Sin comunicacion, diria Niklas Luhmann, no puede hablarse de sistema social, ya que "todo lo que es comunicación es sociedad". La acción y la comunicacion estan intrinsecas en las interrelaciones, y la sociedad puede ser percibida como un conglomerado que se constituye en la comunicación y se instaura entrelazada en la cultura, de la cual los seres humanos se hacen dependientes.

En este sentido sociedad y cultura deben su existencia a las acciones y retroacciones instituidas por la comunicacion. La vida social, tal como lo sugiere Bateson (1984), puede ser comprendida como una estructuración de las "relaciones comunicativas establecidas en el seno de los colectivos humanos y entre estos y su 


\section{IngidNeder Donaire}

Acercamiento transdisciplinario a las realidades complejas e interconectadas a partir de la teoria general de sistemas,

artículo de reflexión

entorno", y. por tanto, la cultura funge como principio organizador y dinamizador de la experiencia humana.

A r recapitularel devenir delateoríadelossistemas, lasteoríasdelacomunicación, la cibernetica, la mecanica cuantica, la relatividad, los procesos de autoorganizacion biológica y las ciencias humanas contenidas en las ciencias de la compleiidad, irrumpen con preponderancia los siguientes principios que se complementan y permiten percibir el mundo de las reaalidades complejas e interconectadas:

El principio de indeterminación de la mecánica cuántica, dado que la posición y la velocidad de un objeto no pueden ser medidos al mismo tiempo, el momento de un observable cambia con respecto al observador, y por lo tanto, la medida exacta de un objeto es incierta y el observador está siempre incluido en lo observado.

El principio de la complementariedad, basado en la correspondencia y como extension de la indeterminacion, considera que los distintos lenguajes posibles y los diferentes puntos de vista sobre el sistema son complementarios, es decir, no existe la posibilidad de un único punto de vista de la reali idad.

El principio del orden a través del caos se refiere al concepto de orden valiéndose de la fluctuacion, y considera que los sistemas evolucionan por medio de saltos discontinuos hacia nuevas organizaciones logradas por medio de fluctuaciones dentro del sistema. Aśl, la transformación discontinua de los sistemas se debe a la amplificacion impredecible de al guna de sus fluctuaciones.

La segunda ley de la termodinámica con su concepto de entropía, que considera que la energla no se transforma sino que se gasta en el proceso de transformacion, por lo que existe una tendencia creciente hacia la degradacion de la energia y a un maximo desorden.

La conjunción de todos estos principios deriva en una epistemología sistémica inmersa en el pensamiento complejo, la cual gula el repensar desdel a multiplicidad y heterogenidad de la realidad, a partir de lo cual se puede percibir y captar lo efimero, 10 novedoso y lo contingente. Es por ello que este repensar se refiere a un proceso del conocer, construir y, mantener un mundo basado en la experiencia, mediado por las interaciones recíprocas y las relaciones cotidianas, donde se permita una configuración permanente de la realidad y su entorno en un ciclo autorregulativo y autogenerativo.

Fundamentos onto-epistémicos de la trama teórico-conceptual de las realidades complejas e interconectadas

En la contemporaneidad, el efecto de red de redes, fundado en la relación multidimensional de la dinámica elemento-sistema de cualquier realidad, ha modificado y ampliado la vision sobre las diversas posibilidades que se expresan 
Revista de

investigaciones UNAD

Volumen 10. Número 1. Junio 2011

en el devenir organizacional mediante la comunicación, pero al mismo tiempo, de esta diversidad surgen ideas y sentimientos que se entretejen en este acontecer, los cuales se pueden vislumbrar desde dos perspectivas:

Por un lado, es producto de la más alta modernización, de la convergencia de tecnologias y de las implicaciones cultural es, que esto ha significado, y por el otro, confiereel manejo de una nueva epistemologia para comprender las interrelaciones que subyacen a esta realidad, las cual es necesariamente se han de concebir desde el conjunto de la sociedad hacia ella misma, y potencialmente, desde esa unicidad a cualquier punto u orden social que se vincule con cualquier otro; segun M orin (2002), esto se observa mediante la interaccion y la reciprocidad en las redes.

Desde esta perspectiva, la ligazón que se produce en el ecosistema por la convergencia de tecnologias y la movilidad discursiva, recrean configuraciones y procesos que dejan de estar segmentados y separados para constiturirse en un devenir que se organiza permanentemente mediante una espiralidad reflexiva de orden emergente generada por las autonomias y dependencias que surgen por la confluencia reticular de flujos y reflujos de saberes.

De ahí que cualquier coexistencia o cofiguración en red busca potencializar el diálogo de saberes y los procesos de conocimiento que se desprenden de este, acordes con las necesidades sentidas del entorno social, cultural, tecnologico, economico y politico, que le dan origen multidimensional mente en lo local-global.

El argumento planteado se refiere primeramente a la superación de las visioness instrumental istas de los medios y a a consideracion de la comunicacion como teoria para la cult turización, es decir, los medios dejaron de ser vistos como un "canal que transmite informacion", en correspondencia con lo que plantea M artín Barbero (1998). Según el, los medios pasan a ser considerados un espacio de encuentro y construccion de influjos sociales, un lugar donde se expresan estrategias de acuerdos en consenso, efectos de dominacion y tacticas de resistencia, y donde tambien se construyen identidades colectivas.

En esta línea de reflexión se recrea un proceso de hipermediación tecnológica, que segun Scolari (2008) permite pasar "de los medios a las hipermediaciones", perdiendose de esta manera la particularidad de objeto, para ganar, en la interrelacion de interactividades y procesos. Por ello, al hacer referencia a la hipermediacion tecnologica, no corresponde a un producto, una herramienta o un medio, sino a un conjunto de "procesos de intercambio, produccion y consumo simbolico que se desarroll an en un entorno caracterizado por una gran cantidad de sujetos, medios y lenguajes interconectados tecnológicamente de manera reticul ar entresi". ( $p .113$ ).

Esteplanteamiento llevaal nacimiento denuevas configuraciones quesurgen por la autorregulacion de actividades y procesos que definen las realidades, asi como por 


\section{IngidNeder Donaire}

Acercamiento transdisciplinario a las realidades complejas e interconectadas a partir de la teoria general de sistemas,

artículo de reflexión

su autoconformación organizacional, quevan más alláde los medios tradicionales. A unado al escenario que presenta la hipermediación tecnologica, otro aspecto a tomar en cuenta es el hecho de que actualmente asistimos a una masificación de la informacion, que conlleva una fragmentacion de las identidades. Tampoco se,puede pasar por alto lo que concierne al manejo y deformacion informativa, así como lo que esto produce en su ambito; de ahí que estas y otras diferencias estructuran este devenir, el cual deberia ser percibido como un universo con mas continuidades que rupturas.

Por este motivo, las realidades interconectadas tienen su fuente y origen en la vinculacion comunicativa, como proceso generador de interrelaciones e interacciones de los contextos que la proveen. Inmersos en esta reflexión, existen a su vez, procesos retroactivos y recursivivos que se producen y complementan en una dinámica dialógica que segun M. orin (2002), permite en un mismo espacio de pensamiento, la coexistencia de lógicas antagonicas, las cuales se necesitan para su produccion y coexistencia.

Este argumento permite abordar la complejidad de estos aconteceres y.presentar la oportunidad de dilucidar, expandir, transformar y reinventar el juego del conocimiento, ya que es posible aprovechar las vinculaciones que prevé la configuracion reticular para que las diferentes areas del saber y las practicas humanas se afecten demultipl es formas y seco-construyan multidireccionalmente, tal como refieren Finquelievich y Finquelievich (2007).

Este ecosistema permite pensar en red, lo que implica tener en cuenta el al to grado de interconexion de los fenomenos y el establecimiento de rutas de encuentros y de reenvios, los cuales trazan recorridos en tramas que fijan los itinerarios de conocimiento colaborativo y espontaneo, en virtud de las diversas formas de la experiencia humana y sus diversas articulaciones en la usabilidad cotidiana, aspecto que envuelve un torbellino generador y transformador de saberes que se funda pertinentemente con su entorno, porque es producto e insumo de este.

A hora bien para comprender mejor lo que se plantea en este estudio como comunicacion intrinseca al entramado social y, lo que refleja la movilidad discursiva, mediante la hipermediacion tecnologica, se recurre a Luhmann (1998), quien considera que "los sistemas sociales consisten, no en hombres, ni en acciones, sino en comunicaciones", ya que un devenir de esta naturaleza, se realiza siempre que se dé una conexión autopoiética de comunicación oportuna con su entorno.

En esta consideración, se capta la dialógica permanente que involucra el proceso comunicacional y su fluir, mediante un torbellino cognitivo generador de habilidades y competencias, apoyado en la complementariedad reciproca que presupone el compartir ideas, pensamientos y experiencias, mediante la movilidad discursiva. 


\section{Revista de investigaciones UNAD \\ Volumen 10. Número 1. Junio 2011}

Este proceso emergente, dinámico y permanente, tal como lo sostiene Martín Barbero (2001), rebasa los límites que tradicionalmente imponían los géneros y medios, para dar, origen a un entramado que se estructura en el lenguaje, el cual tiene como proposito, en primera instancia, admitir los antagonismos que surgen de cada realidad y por ende, generar estados que se complementan de manera concurrente y recursiva, y por otra, reqular la satisfaccion de sus requerimientos y lograr su autoconformacion, si persisten las demandas y desafios que tenga que atender, configurando continuamente entramados reticulares de orden complejo.

\section{Referencias bibliográficas}

Barbero, J . (2003). De los medios a las mediaciones. Bogotá, Colombia: Gustavo Gili.

Bateson, G. (1984). Comunicación. Barcelona, España: Paidós.

Delius, C. y otros (2005). Historia de la filosofía. Desde la antigüedad hasta nuestros días. Barcelona, España: Konemann.

Finquelievich, D. y Finquelievich S. (2007). Más allá de la conectividad: El rol pionero del Estado ante los ciudadanos en la sociedad de la información y el conocimiento. Buenos Aires, Argentina: Universidad de Buenos Aires.

Foerster, H. (1991). Las Semillas de la Cibernética. Buenos Aires, Argentina: Gedisa.

Jutoran, S. (1994). El proceso de las ideas sistémico-cibernéticas. Sistemas familiares, 10 (1), 9-27. Buenos Aires, Argentina.

Luhmann, N. (1998). Sistemas Sociales: Lineamientos para una teoría general. Barcelona, España: Anthropos.

Maruyama, M. (1963). The second cybernetics: deviation-amplifying mutual causal processes. American Scientist, 51, 164-179. Chicago IL, EE. UU.

Maturana, H. (1988). Conversando con Humberto Maturana. Seminario organizado por el Instituto de Terapia Sistémica en Buenos Aires, Argentina.

Maturana, H. (1997). De máquinas y seres vivos, Autopoiesis de la organización de lo vivo. Santiago de Chile, Chile: Universitaria.

Morin, E. (2001). El Método 1. La naturaleza de la naturaleza. Madrid, España: Cátedra.

Morin, E. y otros. (2002). Educar en la era planetaria. Barcelona, España: Gedisa. 


\section{IngidNederDonire}

Acercamiento transdisciplinario a las realidades complejas e interconectadas a partir de la teoria general de sistemas, artículo de reflexión

Scolari, C. (2008). Hipermediaciones. Elementos para una teoría de la comunicación digital interactiva. Barcelona, España: Gedisa.

Sears, F. y Zemanski, M. (1971). Física. Madrid, España: Juan Bravo Gala.

Shannon, C. y Weaver W. (1949). A Mathematical Model of Communication. Urbana, IL, EE. UU.: University of Illinois Press.

Valera, F. (2003). Autopoiesis y biología de la intencionalidad. Santiago de Chile, Chile: Dolmen.

Watzlawick, P. (1981). La realidad inventada. Buenos Aires, Argentina: Gedisa.

Watzlawick, P. y otros. (1990). La realidad inventada. ¿Cómo sabemos lo que queremos saber? Barcelona, España: Gedisa.

Winkin, Y. (1984). La nueva comunicación. Barcelona, España: Kairós. 